\title{
CAPÍTULO XXXIX
}

\section{CULTURA, IDENTIDAD E INNOVACIÓN ANTE LA SOSTENIBILIDAD ARTESANAL}

\author{
Emily Lugo Hernández \\ Magister en desarrollo y cultura, Corporación Universitaria Antonio José de \\ Sucre. Correo electrónico: Docente_investigador4@corposucre.edu.co.
}

\section{Netty Consuelo Huertas}

Doctora en Turismo, Derecho y Empresas. Universidad Tecnológica de Bolívar. Correo electrónico: nhuertas@utb.edu.co. https://orcid.org/0000-0003-38548058

\section{Yuneris Muñoz Cardona}

Magister en Administración de Empresas Turísticas. Universidad Tecnológica de Bolívar. Correo electrónico: ymuñoz@utb.edu.co.

\section{Resumen}

La región Caribe colombiana es reconocida por su diversidad cultural y la presencia de grupos indígenas, cuyas costumbres y actividades económicas generan gran valor patrimonial al territorio; pero que a su vez son caracterizadas por sus niveles de pobreza; hecho que motivó al proyecto a la búsqueda de una propuesta para el desarrollo sostenible de las asociaciones de artesanos de la Región Caribe; considerándolos como representantes de estas culturas y en peligro de extinción. El proyecto se fundamentó en una metodología participativa con representantes de las asociaciones de cada departamento; quienes a través de grupos focales y talleres evaluaron su posición frente a los retos del mercado global y la sostenibilidad no solo de sus actividades económicas, sino de sus comunidades e identidad. Dentro del análisis los artesanos manifestaron que la sostenibilidad de sus comunidades está en riesgo por la poca valorización de sus tradiciones; la entrada de mercancías industrializadas que replican sus bordados, texturas y son comercializadas por valores económicos inferiores; en relación a la penetración de otras culturas manifestaron verlas como oportunidad para la dinamización de sus productos y reconocimiento de estos. Finalmente, se concluyó que para la sostenibilidad se hace necesario la formación y fortalecimiento de las asociaciones en la cadena de exportación, comercialización de sus artesanías; así como la institucionalización de cursos que promuevan el rescate del valor cultural y de identidad de estas culturas. De igual forma, es pertinente la intervención e inversión del estado en la generación de materia prima al alcance de la comunidad y el saneamiento de necesidades básicas.

Palabras clave: artesanías, identidad, sostenibilidad.

www.doi.org/10.47212/tendencias2020vol.xii.40 


\title{
CULTURE IDENTITY AND INNOVATION IN THE FACE OF CRAFTSMANSHIP SUSTAINABILITY
}

\begin{abstract}
The Caribbean region, Colombia is recognized for its cultural diversity and the presence of indigenous groups, whose customs and economic activities generate great heritage value to the territory; but which in turn are characterized by their levels of poverty; a fact that motivated the project to search for a proposal for the sustainable development of artisan associations in the Caribbean Region; considering them as representatives of these cultures and in danger of extinction. The project was based on a participatory methodology with representatives of the associations of each department; who through focus groups and workshops evaluated their position in the face of the challenges of the global market and the sustainability not only of their economic activities, but also of their communities and identity. Within the analysis, the artisans stated that the sustainability of their communities is at risk due to the low value of their traditions; the entry of industrialized goods that replicate their embroidery, textures and are traded for lower economic values; In relation to the penetration of other cultures, they stated that they saw them as an opportunity for the dynamization of their products and their recognition; Finally, it was concluded that for sustainability, the formation and strengthening of associations in the export chain, marketing of their crafts is necessary; as well as the institutionalization of courses that promote the rescue of the cultural value and identity of these cultures. Similarly, the intervention and investment of the state in the generation of raw material available to the community and the sanitation of basic needs is relevant.
\end{abstract}

Keywords: crafts, identity, sustainability.

\section{Proyecto de investigación}

La ponencia y documento presentado es resultado del proyecto titulado: Desafíos de las asociaciones de artesanos de la región Caribe una propuesta para el desarrollo sostenible; financiado por Consejo Profesional de Administración de Empresas - CPAE y realizado por investigadores de las facultades de Administración de Empresas de la Universidad Tecnológica de Bolívar y la Corporación Universitaria Antonio José de Sucre. El presupuesto global del proyecto fue de $\$ 115.650 .000$.

\section{Introducción}

La creación, el conocimiento y la innovación se han convertido en campos centrales de las dinámicas mundiales en actualidad, dado el papel crucial que juegan estos 
elementos en la economía de los países, otorgando un importante lugar a las actividades culturales que se constituyen como un fenómeno económico de relevancia, que moviliza recursos, genera riqueza, empleo a las comunidades y a su vez permiten el desarrollo armónico de los pueblos al considerar la cultura como factor de cohesión, integración y bienestar social (Stolovich, 2002; Weber, 2011).

La cultura es una herramienta potencial en la generación de formas de producción de valor y de comercialización de bienes y servicios que obtiene un significado agregado al obedecer esencialmente a las evoluciones generacionales; la base para la consolidación de los modos de vida, los derechos fundamentales del ser humano, los sistemas de valores, las tradiciones y las creencias (Chantepie, 2011; Terry, 2011). Esta se centra en lo local y es producida especialmente por las dinámicas sociales de una comunidad o territorio tras la gran diversidad de experiencias históricas, la defensa de su espacio y las distintas interpretaciones que han posibilitado la construcción de referentes de identidad.

La identidad de un sujeto social, está dada por el desempeño de distintas acciones en espacios en que el individuo interactúa, perfilando su identidad a partir de referentes como el lugar en donde nació, las relaciones familiares y sociales asociadas a su desarrollo como persona, las labores desempeñadas para el mantenimiento familiar, el entorno escolar, los niveles de religiosidad, relaciones laborales y productivas que proporciona cierto nivel de cohesión social y con este un sentido común de pertenencia a algún sitio y a un colectivo específico, idea que da sentido a la forma en que interpretan y generan sus representaciones como grupo (Contreras, 2013).

No obstante, un individuo no se relaciona a lo largo de su vida con un solo espacio, comunidad o colectivo social que cree una identidad única o estática en el tiempo (Altomare y Seoane, 2008) hecho que al contraponerse con fenómenos como la globalización, apertura de mercados y conectividad genera nuevos dinamismos y características propias en los individuos permeando de esta manera las culturas locales específicamente la creación, el conocimiento y los rasgos identitarios que podrían definir a estas. 
La penetración de otras culturas genera en lo local, grandes riesgos sociales y económicos relacionados a la posición de debilidad en los mercados o influencia de estas en las tradiciones propias de una región, evidenciados en la tenencia de alto consumo de productos extranjeros y la desaparición de empresas locales al ser remplazadas por multinacionales (Organización de las Naciones Unidas para la Educación, la Ciencia y la Cultura (UNESCO, 2011), así como la desaparición de aquellos rasgos propios como dialectos; técnicas de producción y saberes de comunidades aborígenes que han sido desplazados por el saber científico y tecnológico; y culturalmente han perdido su carácter o reconocimiento como comunidades indígenas o tradicionales de un territorio (Mordini, 2007).

Ahora bien, sumado a lo anterior, en siglo XXI el desarrollo sostenible ha tomado gran relevancia para los territorios y el mercado empresarial. Este es concebido como un proceso integral, para alcanzar un desarrollo económico, social y cultual, que conserve, enriquezca y promueva en vez de destruir las bases en las que inevitablemente se asienta y desenvuelve la actividad humana y tiene como objetivo administrar de forma eficiente y racional los recursos disponibles, de tal manera que sea posible mejorar la calidad de vida de las generaciones futuras, con el desafío de transformar los patrones de producción y consumo de la sociedad (Aguilar, 2003).

De esta forma, la innovación, la globalización, el desarrollo sostenible, la apertura de mercados se han convertido más que en barreras, en retos para los territorios y empresas fomentando la implementación de nuevas estrategias para generar valor en sus productos, procesos y contextos con un enfoque sustentable a través de una cultura que valore la creatividad e innovación (Ahme et al., 2012).

Dentro del sector cultural una de las actividades que más sobresale bajo estos retos y promoción de estrategias basadas en la cultura y fomentadora de creatividad e innovación, es la artesanía, al reunir sus productos un conjunto de características que conforman el patrimonio y la identidad cultural de las comunidades y pueblos, vinculando tradición e innovación, lenguajes y estéticas, según los diferentes períodos históricos. Vectores de diversidad cultural, hechos por artesanos y artesanas que han trasmitido sus saberes de generación en generación, contribuyendo a un rico patrimonio. 
Colombia es reconocida por su compleja y rica diversidad cultural que se expresa en una gran pluralidad de identidades y de expresiones culturales de los pueblos y comunidades que la conforman; esta es considerada como el patrimonio más valioso de la nación. Afrocolombianos, raizales, palenqueros, rom o gitanos, pueblos indígenas, comunidades campesinas, mestizos, y comunidades originadas en migraciones externas enriquecen el mosaico cultural del país; especialmente el de la región Caribe donde se entrelazan todas estas culturas y en conjunto con su riqueza biodiversa favorecen en esta región la producción artesanal.

No obstante, de acuerdo con la información de Artesanías de Colombia, los hogares pertenecientes al sector artesanal se caracterizan por ser pobres $(60 \%)$, estar ubicado en su gran mayoría en áreas rurales, y conformados por población vulnerable (52\%); de igual forma esta actividad es realizada generalmente por mujeres, a menos que esta vincule el uso de materiales como la madera y el metal; y habitualmente los artesanos y artesanas se encuentran organizados en Asociaciones, las cuales deben enfrentarse a las dinámicas de un mercado globalizado y competitivo, lo que crea la necesidad de fortalecer las condiciones empresariales de estas asociaciones para contribuir al desarrollo empresarial; pero sin afectar el patrimonio inmaterial que constituyen las artesanías y la gestión del conocimiento que permite que estas se gesten.

El presente documento, presenta la reflexión derivada del proyecto: Desafíos de las asociaciones de artesanos de la región Caribe. Una propuesta para el desarrollo sostenible y busca evaluar los retos que presentan las asociaciones de artesanos de la región Caribe para crear una sinergia entre la cultura, identidad de sus comunidades, la incorporación de la innovación a sus productos y la sostenibilidad de su arte y oficio artesanal.

\section{Fundamentación teórica}

\section{Cultura}

La cultura es el bien común que las comunidades y sociedades han de incorporar en la configuración de sus futuros y en la lucha contra las dificultades que genera la pobreza y la desigualdad, es una dimensión importante en la evolución del desarrollo y su función radica en el papel político y educacional que esta aporta en la consecución 
del bienestar colectivo (Martinell, 2010). Es a través de la cultura que las civilizaciones se forman como perfecta y única, creando autonomía y limitando su continuación a otra civilización (Abello et al., 2000).

La cultura es el conjunto de los rasgos distintivos espirituales y materiales que caracterizan a una sociedad, que abarca, además de las artes, las letras, los modos de vida, las maneras de vivir juntos, los sistemas de valores, las tradiciones y las creencias" (UNESCO, 2001) ese conjunto de expresiones diversas, mediante las cuales una sociedad moldea y reflexiona su convivencia, incluyendo las imágenes e ideas a través de la cual dicha sociedad representa las formas en que convive y quiere convivir (Programa de las Naciones Unidas para el Desarrollo (PNUD, 2020) el cuarto pilar que moldea lo que se concibe como desarrollo y determinar las forma de actuar de las personas en el mundo (UNESCO, 2010).

\section{Identidad}

La identidad constituye el eje central de reconocimiento de una cultura, un pueblo, o una nación (Abello et al., 2000) es el conjunto de prácticas sociales que involucran simultáneamente a cierto número de individuos o grupos, exhiben características morfológicas similares en la contigüidad temporal y espacial, implican un campo de relaciones sociales, así como también la capacidad de la gente involucrada para conferir un sentido a lo que está haciendo o va a hacer; la identidad define la capacidad para la acción autónoma así como la diferenciación del actor respecto a otros dentro de la continuidad de su identidad (Giménez, 2005).

La identidad se constituye como el sentido de pertenencia a una colectividad, a un sector social, a un grupo específico de referencia; en este sentido la identidad cultural de un pueblo estará definida por aspectos como la lengua, las relaciones sociales, ritos y ceremonias propias, o los comportamientos colectivos y los sistemas de valores y creencias de una comunidad. La identidad cultural solo existe por la memoria, por la capacidad de reconocer el pasado, los elementos simbólicos o referentes que le son propios y que ayudan a construir el futuro. 


\section{Innovación}

La innovación es la incorporación de conocimiento a los procesos productivos y sociales, un determinante de la competitividad y el crecimiento, dado que permite obtener nuevos procesos, productos y servicios, que al transferirse al mercado y la sociedad modifican la capacidad de competencia de los productores; tomando en cuenta que la fuente de toda mejora de competitividad es la acumulación de capacidades a nivel de organizaciones.

La innovación es la introducción de un nuevo o significativamente mejorado de un bien o servicio, proceso o método implementado en las prácticas de una comunidad u empresa (Mercado, 2017). La innovación brinda ventajas competitivas sostenibles a las organizaciones al poderse dar en cualquier actividad de la cadena de valor de la empresa, ya que esta provee de nuevos o mejores fundamentos para competir, manifestándose sea en un nuevo diseño de producto, un nuevo proceso de producción, un nuevo enfoque de marketing, o una nueva forma de llevar a cabo la organización (Porter, 1990).

Laactividad innovadora está íntimamente ligada con la productividad y competitividad de las empresas, es un elemento estratégico del negocio y factor clave para garantizar la supervivencia de estas en un mercado cada vez más global y competitivo; de esta forma la innovación es un elemento que da garantía, la consolidación y crecimiento en los mercados locales de las organizaciones y en su proyección hacia los mercados internacionales (Zapata, 2017).

\section{El producto: artesanías y sus características}

La artesanía es un tipo de actividad en la que se involucran elementos económicos, técnicos, productivos, comerciales, sociales, estéticos y culturales de gran complejidad y se desarrolla integrada con otras expresiones del arte y la cultura y con la propia producción industrial. (Benitez, 2009). Estos productos artesanales se caracterizan por (Unesco, 2017): son elaborados por artesanos, quienes trabajan totalmente a mano (esta contribución manual directa es el componente más importante del producto acabado) o con la ayuda de herramientas manuales o incluso de medios mecánicos; no existen límites en cuanto a la utilización de materias primas procedentes de recursos 
sostenibles; estos productos se basan en sus características distintivas, que pueden ser utilitarias, estéticas, artísticas, creativas vinculadas a la cultura, decorativas, funcionales, tradicionales, simbólicas y significativas religiosa y socialmente.

Las artesanías satisfacen diversos tipos de necesidades que se asocian con otras expresiones culturales como las fiestas populares, el diseño y las artes visuales. Los compradores pueden utilizar las artesanías para el autoconsumo individual o colectivo, otros para comercialización, bien sea para el mercado turístico e incluso para el mercado internacional, a través de la exportación.

Los productos artesanales constituyen también una forma de conocimiento y de comunicación que perpetua valores culturales entre diversas generaciones. Se asocian con formas de consumo cultural diferenciadoras de los estándares del mercado de objetos industriales, se pueden mencionar consumidores coleccionistas, aficionados a la artesanía y el arte (Benitez, 2009).

\section{Sostenibilidad artesanal}

La sostenibilidad exige la integración de factores económicos, sociales, culturales, políticos y ecológicos y la articulación constructiva de los criterios que abordan el desarrollo (Gallopín, 2003) esta busca del bienestar de los seres humanos a través del tiempo, relacionado a una buena vida; que depende de las habilidades y oportunidades de los individuos y las sociedades, de adquirir condiciones de vida, de forma que contribuyan a la preservación de los recursos económicos y ambientales para futuras generaciones (Lugo, 2018).

Por su parte, al hablar de artesanal (artesanías), se hace referencia al conjunto de conocimientos que han sido heredados y responde a las necesidades de las comunidades, el soporte simbólico de costumbres, creencias y rituales, la forma de vida que adquiere y reproduce una comunidad. Las técnicas artesanales corresponden a lo material del patrimonio inmaterial, son importantes en la preservación del patrimonio cultural al fundamentar la cosmovisión de los pueblos (Ministerio de Cultura (MINCULTURA), 2015). 
En este sentido la sostenibilidad artesanal involucra la articulación y preservación de la manifestación cultural que deja la actividad, las artesanías y ese conocimiento y técnicas ancestrales transmitidas de generación en generación que permiten la creación de piezas de identidad de una comunidad y el reconocimiento autónomo de estas.

\section{Metodología}

La metodología utilizada fue de corte cualitativo, con un enfoque descriptivo y un método participativo, cuya fuente de información principal fue primaria aportada por los representantes de las asociaciones de cada departamento de la región Caribe, Guajira, Cesar, Magdalena, Sucre, Córdoba, Atlántico y Bolívar, quienes a través de entrevistas evaluaron su posición frente a los retos del mercado global y la sostenibilidad de su actividad económica, comunidades e identidad.

Para realizar la investigación, se abordaron cuatro fases, la primera fue la etapa de revisión documental que permitió identificar desde la literatura la competitividad de la industria artesanal en la región Caribe retos de las organizaciones artesanales frente a la sostenibilidad, posteriormente se realizó un barrido por los municipios artesanales, reconocidos en los departamentos, en los que se identificaron las artesanías representativas y las asociaciones que se dedicaban a la actividad. Seguidamente, se realizó la recolección de la información y análisis de la misma que permitió caracterizar las asociaciones de artesanos y alcanzar la consecución de los objetivos propuestos en la investigación.

\section{Resultados}

La Artesanía son el resultado de la creatividad y la imaginación, plasmado en un producto en cuya elaboración se ha transformado racionalmente materiales de origen natural, generalmente con procesos y técnicas manuales; los objetos artesanales van cargados de un alto valor cultural y debido a las técnicas y su proceso de elaboración son considerados como piezas únicas, parte de la identidad y de la creatividad de las personas (Rivas, 2018). 
En Colombia, la actividad artesanal es parte sustancial del desarrollo económico, social y cultural del país; su contribución en la generación de empleo e ingresos por artesano es en promedio 1, 2 puestos de trabajo, contribuyendo el 15\% del empleo de la industria manufacturera nacional. Su influencia en el rescate y enriquecimiento de la cultura es clave para el desarrollo y fortalecimiento de la identidad, así como la asociatividad para la cohesión social de los territorios (Benítez, 2013).

La región caribe colombiana, reúne una de las ofertas artesanales más representativas de nuestro país; focalizada especialmente en los departamentos Córdoba, Sucre, Bolívar, Atlántico y la Guajira donde se destacan productos emblemáticos como el sombrero vueltiao, las mochilas Wayúu y las máscaras del tradicional Carnaval de Barranquilla. Los artesanos de esta región se encuentran organizados principalmente en asociaciones y cooperativas; su trabajo gira entorno a la familia, hecho que facilita la transmisión de saberes de generación en generación.

El sector artesanal a nivel nacional es administrado por el Gobierno Nacional bajo las directrices de Artesanías de Colombia, quien promueve la cooperación y promoción de buenas prácticas, buscando que los artesanos que tienen más experiencia en determinados saberes puedan orientar a aquellos grupos que están iniciando; lo que hace que este sector crezca de manera organizada y con una visión de crecimiento integral y sostenible. De esta manera, al analizar la competitividad de la industria artesanal encontramos que en torno a la rivalidad entre los competidores existente una amenaza de productos o servicios sustitutos, dado que entre los distintos departamentos se ofrecen productos con utilidad o funcionalidad similar los artesanos podrían competir entre sí; pero que por sus principios de cooperación reúnen esfuerzos para trabajar en conjunto lo que podría ser considerado una ventaja competitiva del sector; sin embargo dada la apertura económica y los diferentes tratados internacionales e industria manufacturera desarrollada en el país los productos artesanales en la región en algunas ocasiones se encuentran expuestos a un riesgo calidad/precio lo que podría representar una amenaza para la sostenibilidad artesanal.

Por su parte al analizar el Poder de negociación de proveedores fue posible denotar que en relación a los proveedores de materias primas que necesitan los artesanos de 
la Región Caribe para elaborar sus artesanías, cuando estas proceden de procesos biológicos vegetales el acceso es limitado, ya que la estacionalidad característica de dichos procesos exige a los artesanos asumir técnicas de almacenamiento y adecuación de estas materias primas que incrementa los costos y limita su productividad. Cuando para la elaboración de las artesanías las materias primas son de origen mineral, como oro, plata u otros, el precio y la oferta de mercado resultan ser variables importantes para acceder a estas, como también lo son la capacidad de pago del artesano, ya que condiciona los volúmenes de compra que puedan hacer. Referente a esto último la caracterización arrojó que el $71 \%$ de los artesanos compra al detal siendo el mismo territorio donde habitan el principal medio de abastecimiento, en un $84 \%$, ya que sólo $17 \%$ de los artesanos se proveen de sitios ubicados fuera de su lugar de trabajo.

En cuanto al Poder de negociación de los clientes, destacamos que dada la precariedad económica de las familias artesanas; la relación artesana-cliente, se ve asociada a un eslabón más de la cadena artesanal, los comercializadores de artesanías en el país, representados principalmente por personas naturales que compran y venden el producto artesanal con diferenciales de precio significativos y desventajosos para el artesano. Así mismo, al indagar sobre las Barreras de entrada para el caso de las Artesanías, las barreras de entrada son menores, porque acceder a la materia prima no representa un costo alto, dado que muchos de estos trabajos lo que requieren es tener mano de obra disponible, la cual están dispuestos a colocar los artesanos, y materia prima; en esencia las principales barreras se presentan por el desconocimiento de la técnica a utilizar o el uso de los materiales; no obstante esta barrera se elimina porque el conocimiento se comparte de generación en generación y por cursos que en ocasiones son ofertados por entidades como el SENA. Precisamente por esas bajas barreras de entrada, se requiere que haya una diferenciación del producto y además una protección con la Denominación de Origen, pues hoy por hoy las mochilas Wayuu se están elaborando en diferentes municipios de la Región Caribe, por personas que no pertenecen a la cultura Wayuu y ni pertenecen al departamento de la Guajira.

El análisis de las fuerzas de competitividad contrastado con el factor de innovación encontrado en las artesanías nos permite destacar el papel que juegan los talleres ofrecidos por Artesanías de Colombia de innovación creación, que han permitido la transformación de productos artesanales decorativos en productos utilitarios y han 
brindado oportunidades de apertura en el mercado internacional a muchas familias de artesanos de la región Caribe, en este sentido, es importante destacar el papel que juega el turismo en la comercialización del producto dado que en los lugares donde hay afluencia de turistas la demanda de artesanías es mayor, lo que hace a los artesanos motivarse ante la elaboración de piezas atractivas para ser competitivos en los mercados.

Ahora bien, la innovación y creación de productos nuevos si bien se constituyen en elementos importantes para la sostenibilidad de la actividad económica, han generado debates al hablar de la preservación de las culturas e identidad de las piezas artesanales de la región, al respecto citamos las palabras de los wayuu quienes puntualizaron que " no se puede perder el conocimiento de nuestros ancestros, ni elaborar productos basados en otras culturas, pero es necesario utilizar nuestros saberes en la elaboración de nuevos productos", para resaltar la importancia y retos que constituye la creación de nuevos productos y la preservación de las técnicas y conocimiento transmitido por generaciones de forma consiente y sostenible, muchos artesanos dadas la limitaciones económicos transmiten sus saberes o cambian las técnicas y atributos de estas piezas consideradas patrimonio inmaterial, por complacer al cliente; o comercializan sus productos como un bien más del mercado sin darle el valor que este representa; la transmisión de estos saberes por entidades como el SENA representa una barrera para la sostenibilidad de la cultura identificaría de la artesanía; y la protección de estos elementos por denominaciones de origen, una amenaza para su preservación y competitividad en los mercados.

\section{Conclusiones}

La artesanía en la región Caribe dada su diversidad y complejidad presentan un gran potencial para la economía de la región como actividad económica sostenible; no obstante este potencial se ve empañado por la calidad de vida y acceso a servicios básicos de los hogares artesanos que constituyen el primer pilar de la cadena artesanal; como fortalezas del sector destacamos la originalidad de los productos; la capacidad de innovación de los talleres, la productividad y organización en cooperativas y asociaciones de artesanos encontrados en la región; de igual forma como debilidades al interior de este sector se encontró el acceso a materias primas, los canales comercialización, las condiciones económicas de los artesanos y la no denominación de origen de algunas de las artesanías. 
De esta manera, para la sostenibilidad artesanal, como oportunidad se denotaron el crecimiento del turismo en el país que sirve de vitrina al sector y el apoyo generado por el gobierno nacional a través de la organización Artesanías de Colombia; como amenazas las bajas barreras a la entrada de la actividad; la comercialización de productos industriales de otros países que replican el producto artesanal y el fomento de elaboración de productos por organizaciones externas a las culturas y hogares artesanales.

En torno al debate entre cultura, identidad e innovación; los artesanos de la región Caribe son conscientes que para la preservación de la artesanía y el reconocimiento de sus comunidades como artesanas es necesario mantener la calidad y las técnicas utilizadas por sus ancestros; pero a su vez generar nuevos productos y utilidades de sus técnicas que les permitan entrar al mercado y ser competitivos; en la medida que conservan su tradición como parte de su riqueza cultural inmaterial y esta sea replicada en sus generaciones futuras.

\section{Referencias bibliográficas}

Banco Mundial. (2004). Informe de Desarrollo. Bogota, Colombia.

Abello, I., Zubiría, S. y Sánchez, S. (2000). Cultura: Teorias y Gestión. San Juan de Pasto: Universidad de Nariño.

Aguilar, S. (2003). El principio de integración medioambiental dentro de la Unión Europea: la imbricación entre integración y desarrollo sostenible. Papers Revista de Sociología, 77-97.

Ahmed, P., Shepherd, C. y Ramos, L. (2012). Administración de la innovación. Editorial Pearson,: México.

Altomare, M. y Seoane, J. (2008). Identidad colectiva y clase social. Universitas Humanística, 65.

Benitez, S. (2009). La artesanía latinoamericana como factor de desarrollo económico, social y cultural: a la luz de los nuevos conceptos de cultura y desarrollo. 
Cultura y Desarrollo, 1-19. Obtenido de. http://www.lacult.unesco.org/ docc/CyD_6.pdf

Benítez, S. (2013). La artesanía latinoamericana como factor de desarrollo económico, social y cultural: a la luz de los nuevos conceptos de cultura y desarrollo. Cultura y desarrollo.

Chantepie, P. (2011). Primer Análisis de las crisis y de las políticas culturales contracíclicas en Francia. En El sector cultural Hoy: análisis, desafios y respuesta (págs. 50-65). Cartagena: Universidad Tecnologica de Boliva.

Contreras, G. (2013). Comunidad y cultura. Espacios Transnacionales. Obtenido de http://www.espaciostransnacionales.org/conceptos/comunidadycultur

Gallopín, G. (2003). Sostenibilidad y desarrollo sostenible: un enfoque sistémico. CEPAL.

Giménez, G. (2005). La cultura como identidad y la identidad como cultura. México: Consejo Nacional de la Cultura y las Artes.

Lugo, E. (2018). Lineamientos para el fomento del turismo rural, en clave de desarrollo y cultura, en el municipio de Ovejas Sucre. Cartagena de Indias : Universidad Tecnologica de Bolivar.

Martinell, A. (2010). Cultura y Desarrollo:Un compromiso para la libertad. Madrid : Siglo XXI.

Mercado, C. (2017). Propuesta de modelo de gestión de la innovación para fortalecer la competitividad de las pymes del sector minero de calizas del municipio de Toluviejo-Sucre. Cartagena : Universidad Tecnologica de Bolivar.

MINCULTURA. (30 de 07 de 2015). Nuestro Patrimonio Cultural al alcance de todos. Obtenido de https://www.mincultura.gov.co/areas/patrimonio/mes- 
del-patrimonio/patrimonio-cultural-al-alcance-de-todos/Paginas/NuestroPatrimonio-Cultural-al-alcance-de-todos.aspx

Mordini, E. (2007). La Globalización y la perdida de la Identidad. Obtenido de http:// files.bvs.br/upload/S/1555-8746/2007/vn4/a125-131-2.pdf

Perez, C. y Pizzarro, A. (2016). Competitividad en las organizaciones: las mipymes del sector manufacturero en sincelejo, sucre - colombia. TEACS.

PNUD. (20 de 09 de 2020). Obtenido de Programa De Las Naciones Unidas: http:// unesdoc.unesco.org/images/0014/001440/144076s.pdf

Porter, M. (1990). The Competitive Advantage of Nations. Harvard Business Review.

PROCOLOMBIA. (2016). Agnóstico del Sector Artesanal en Colombia. Bogota.

Rivas, R. (2018). La Artesanía: patrimonio e identidad cultural. Revista de Museología" Kóot”, 80-96.

Rosales, R. (1997). La Asociatividad como Estrategia de Fortalecimiento de las PYMES.

Stolovich, L. (2002). La cultura es capital: entre la creación y el negocio : economía y cultura en el Uruguay. Montevideo. Uruguay: FONA.

Terry, G. (2 de Junio de 2011). Cultura, Identidad Cultural, Patrimonio y Desarrollo Comunitario Rural:Una nueva mirada en el contexto del siglo XXI latinoamericano, en Contribuciones a las Ciencias Sociales. Obtenido de www.eumed.net/rev/cccss/12/

UNESCO. (2001). Conferencia mundial de Politicas Culturales. Declaración de la UNESCO. 
UNESCO. (2010). La cultura es el cuarto pilar del desarrollo. Chicago: Agenda 21.

UNESCO. (2011). Los desafios de la artesanía en los países del Cono Sur: Excelencia y Competitividad. Montevideo, Uruguay.

UNESCO. (2017). http://www.unesco.org. Obtenido de http://www.unesco.org/new/es/ santiago/culture/creative-industries/crafts-design/

Weber, A. (2011). Las ciudades, los territorios y las politicas culturales en tiempos de crisis. En El sector cultural Hoy: oportunidades, desafos y respuestas (pág. 240). Cartagena de indias: Universidad Tecnologia de Bolivar.

Zapata, M. (2017). Planificación Estratégica: Evaluación del ambiente interno de la empresa para la obtención de ventaja competitiva. Managua: Universidad Nacional Autónoma de Nicaragua,. 